\title{
Vascular cognitive impairment in experimental hypertension: the microglial culprit
}

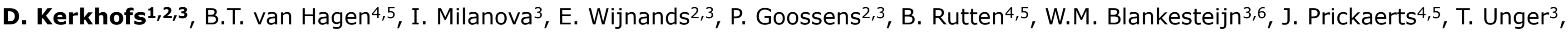
E.A. Biessen2,3, R.J. van Oostenbrugge ${ }^{1,3,5}$, S. Foulquier 3,6

${ }^{1}$ Department of Neurology, Maastricht University Medical Center+; ${ }^{2}$ Department of Pathology, Maastricht University, The Netherlands; ${ }^{3}$ CARIM, School for Cardiovascular Diseases, Maastricht University, The Netherlands; ${ }^{4}$ Department of Psychiatry and Neuropsychology, Maastricht University, The Netherlands; ${ }^{5}$ MH\&NS, School for Mental Health and Neurosciences, Maastricht University, The Netherlands; ${ }^{6}$ Department of Pharmacology-Toxicology, Maastricht University, The Netherlands.

\section{Background}

Hypertension is a major risk factor for cerebral small vessel disease (CSVD) $)^{1,2}$. CSVD is the most prevalent cause of vascular cognitive impairment ${ }^{2}$. We showed before that hypertension induced by a prolonged Angiotensin II (Ang II) infusion in a mouse model was associated with microglial activation, focal blood brain barrier (BBB) leakages, myelin loss, and short-term memory impairment ${ }^{3}$. However, the exact role of microglia in the pathological cascade of CSVD remains elusive.

Objective

In this study, we aim to decipher the contribution of microglia in this pathological cascade by a pharmacological loss-of-function study using a highly selective CSF-1R inhibitor (PLX5622).

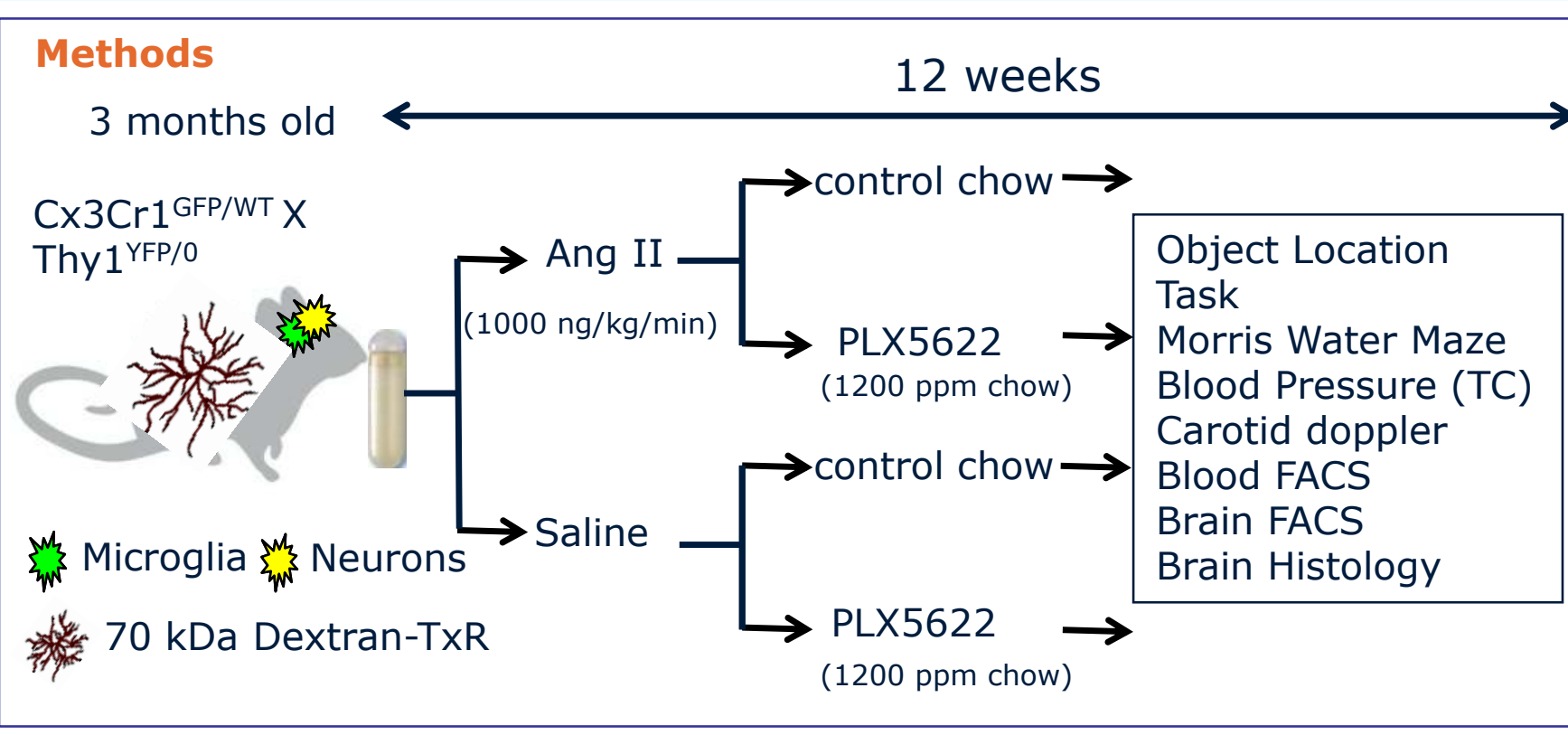

\section{Treatment}

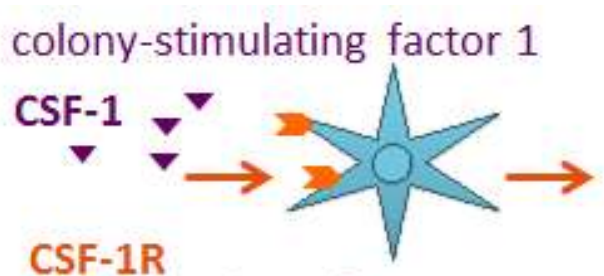

CSF-1R

Colony-stimulating factor 1 receptor
PLX5622 (Plexxikon, Berkeley, CA, USA) inhibitor of CSF1R tyrosine kinase $(\mathrm{KI}=5.9 \mathrm{nM})^{4}$ proliferation $\quad 50$-fold selectivity over 4 related differentiation - >100-fold selectivity against panel of 230 kinases $^{4}$
Results

SBP, heart weight and carotid pulsatility index were increased by AngII and were not affected by PLX5622. Long-term spatial memory was not affected by AngII and/or PLX5622 treatment. Short-term memory was significantly impaired by AngII, and this effect was blunted in AngII mice treated with PLX5622. Histological and FACS analyses confirmed the effective depletion of microglia in PLX5622 treated groups.

\section{Cardiovascular parameters}

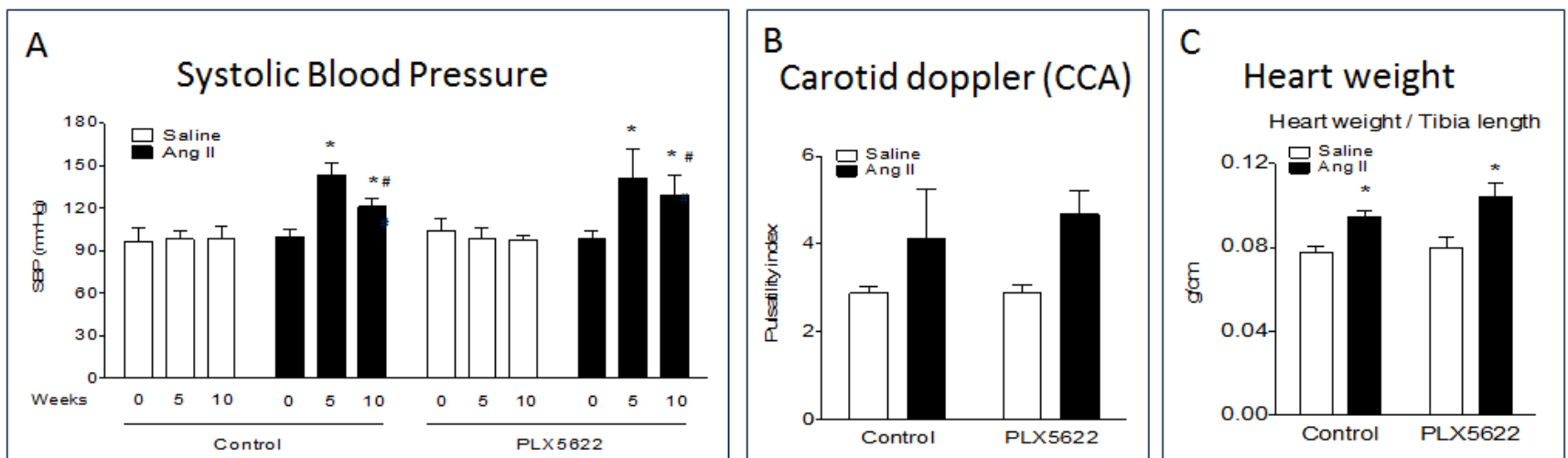

A Systolic blood pressure over the treatment period, 2-W ANOVA $p_{\text {int }}<0.001 ; p_{\text {time }}<0.001 ; p_{\text {AngII }}<0.05$; ${ }^{*}:$ : $<0.05$ vs week 0 ; \#: $P<0.05$ vs week 5 (Tukey post test). B Pulsatility index measured with flow velocity doppler, 2 -W ANOVA $\mathrm{p}_{\text {int }}>0.05 ; \mathrm{p}_{\mathrm{PLX}}>0.05 ; \mathrm{p}_{\text {AngII }}=0.01 * \mathrm{C}$ Cardiac hypertrophy (heart
weight/tibia length), 2 -W ANOVA $\mathrm{p}_{\text {int }}>0.05 ; \mathrm{p}_{\mathrm{PLX}}>0.05 ; \mathrm{p}_{\text {AngI }}<0.001 ; * \mathrm{p}<0.05$ vs Saline (Tukey post test).

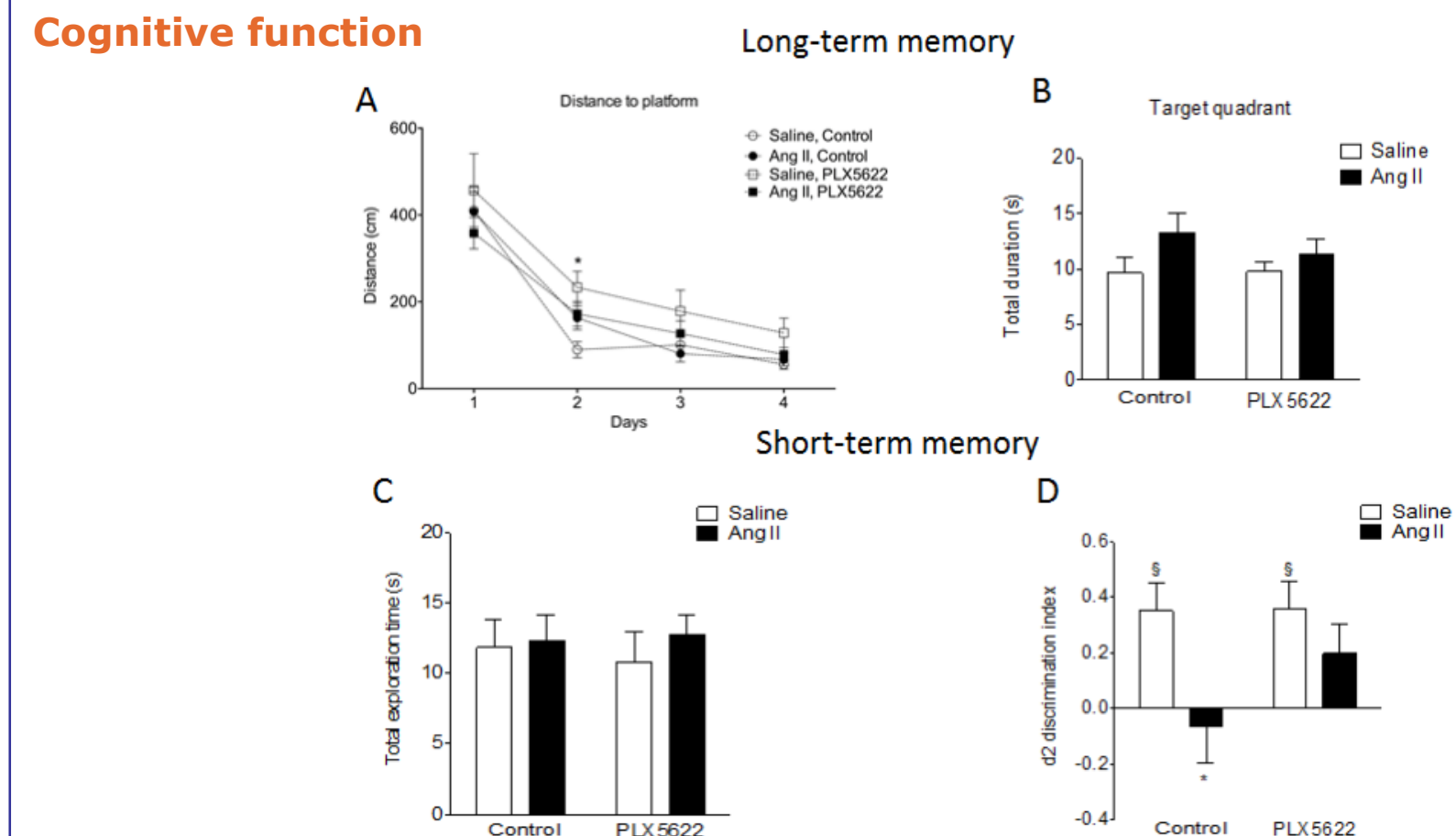

A-B Long-term memory was assessed using Morris Water Maze Task. (A) Learning phase, 2-W ANOVA $p_{\text {int }}>0.05 ; p_{\text {time }}<0.001 ; p_{\text {groups }}<0.05 ; *: p<0.05$ vs control (Tukey post test). (B) Probe trial, $2-W$
ANOVA $p_{\text {int }}>0.05 ; p_{\text {pix }}>0.05 ; p_{\text {Angit }}>0.05$. C-D Short-term memory assessed using Object Location Task. (C) Total exploration time, $2-W$ ANOVA $p_{\text {int }}>0.05 ; p_{\text {pix }}>0.05 ; p_{\text {n }}>0.05$. (D) Discrimination index, 2 -W ANOVA $\mathrm{p}_{\text {int }}>0.05 ; \mathrm{p}_{\mathrm{PIX}}>0.05 ; \mathrm{p}_{\text {AngII }}<0.05$; $*$ : $\mathrm{p}<0.05$ vs Saline (Sidak's multiple comparison post-test); $\S: \mathrm{p}<0.05$ vs $\mathrm{d} 2=0$ (Two tailed t-test)
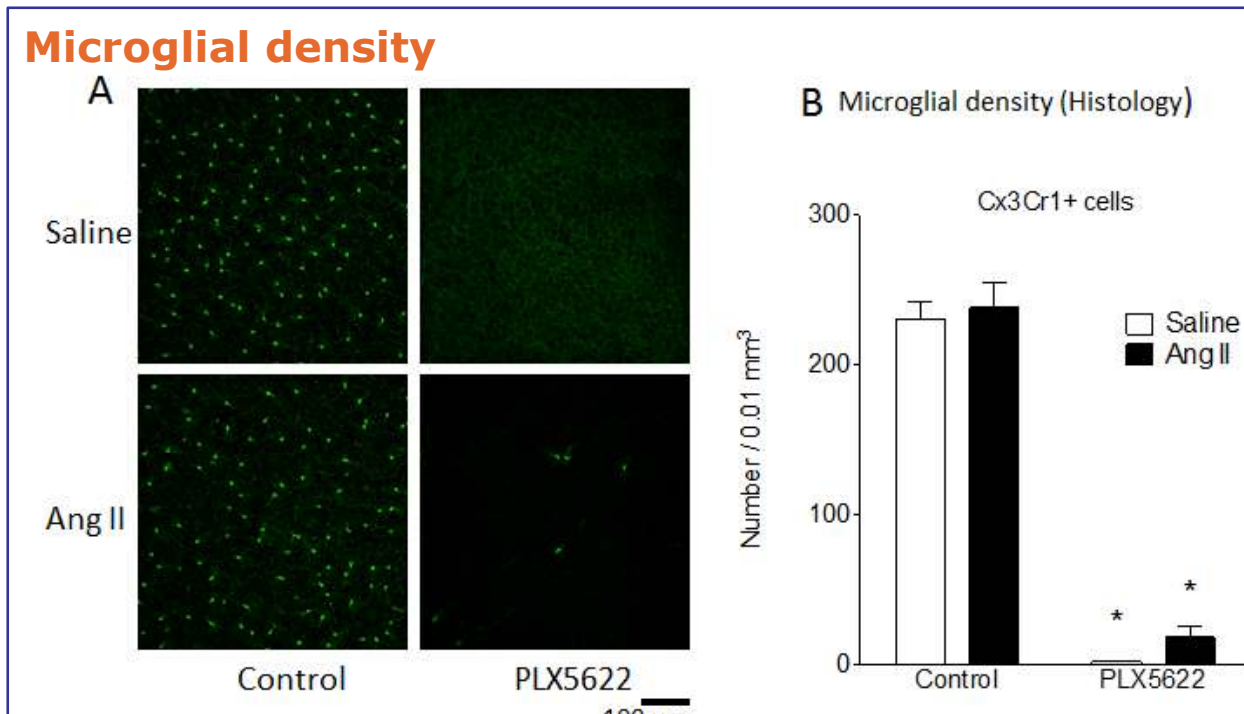

C Microglial density (FACS)

A Representative pictures of $\mathrm{C} \times 3 \mathrm{Cr} 1+$ cells in cortical areas of Control ( $\mathrm{A}$, upper row) and Ang II mice ( $\mathrm{A}$, bottom row), without treatment(left row) and with PLX5622 treatment (right row). B Quantification of the

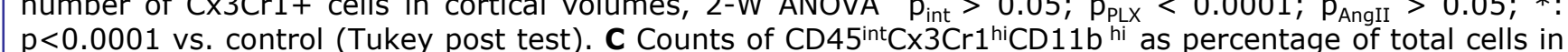
whole brain, 2-W ANOVA $\mathrm{p}_{\text {int }}>0.05 ; \mathrm{p}_{\mathrm{PLX}}<0.0001 ; \mathrm{p}_{\text {AngII }}>0.05 ; *$ : $\mathrm{p}<0.0001 \mathrm{vs}$. control (Tukey post whole
test).

Conclusion

Our study revealed that short-term memory impairment in mice submitted to prolonged ANG II induced hypertension is absent when microglia are depleted, independently of any cardiovascular changes.

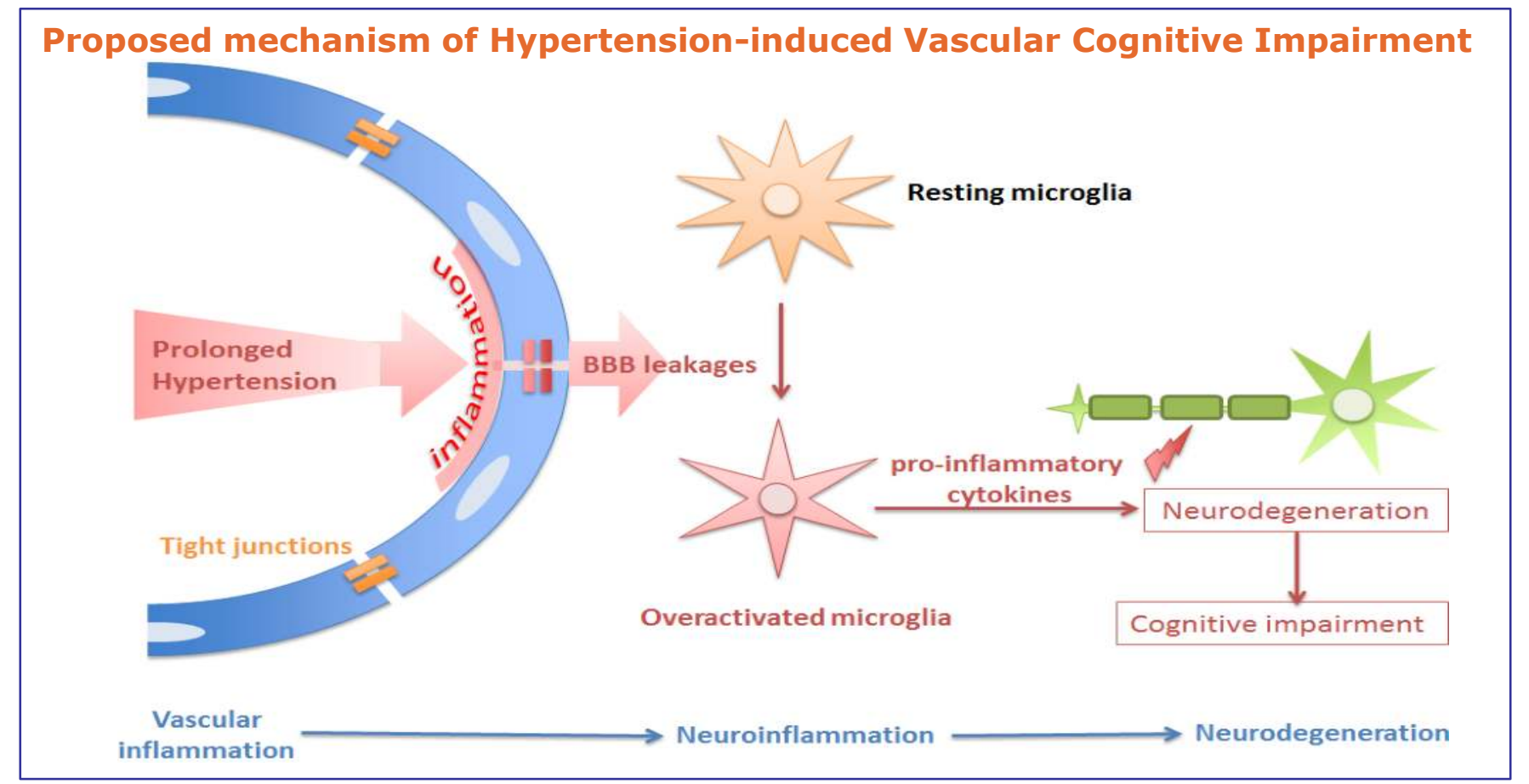

Ongoing

Identifying size and localization of BBB Leakages (70kDa-dextranTxR; IgG) Immune cell phenotyping, including perivascular macrophages (CD206+)

References 1. Pantoni L. Lancet Neurol. Jul 2010;9(7):689-701; 2. Iadecola C. The pathobiology of vascular dementia. Neuron. 2013;80(4):844-66; 3. Foulquier S. Hypertension Res. 2018, accepted; 4. Dagher N. et al. J Neuroinflammation 2015 Aug 1;12:139 Funding EU Horizon 2020 (grant agreement No 666881, SVDs@target

\section{a \\ SVDS}

4 Maastricht UMC+

Maastricht University in Learning!
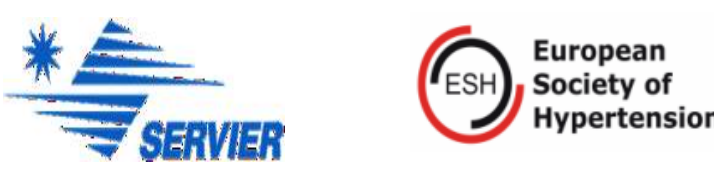

Plexxikon
Dept of Neurology and pathology CARIM-School for Cardiovascular Disease

$\mathrm{T}+31433884895$ F +31433884149
Maastricht University P.O. Box 616 6200 MD Maastricht, The Netherlands 